\title{
TheYale-Brown Obsessive-Compulsive scale: factor structure of a large sample
}

\section{Gideon E. Anholt ${ }^{1}$, Patricia van Oppen ${ }^{1}$, Danielle C. Cath ${ }^{1,2}$, Johannes H. Smit ${ }^{1}$, Johan A. den Boer ${ }^{3}$, Marc J. P. M. Verbraak ${ }^{4,5,6}$ and Anton J. L. M. van Balkom ${ }^{1}$}

\author{
1 Department of Psychiatry and EMGO Institute, VU University Medical Center, Academic Outpatient Clinic for Anxiety Disorders, GGZ inGeest, Amsterdam, \\ Netherlands \\ 2 Altrecht Utrecht and Department of Clinical psychology, Utrecht University, Utrecht, Netherlands \\ ${ }^{3}$ Department of Biological Psychiatry, University Medical Center Groningen, Groningen, Netherlands \\ ${ }^{4}$ Behavioral Science Institute, Radboud University, Nijmegen, Netherlands \\ ${ }^{5}$ ADAPT Expert Center of Anxiety Disorders, Adhesie, Almelo, Netherlands \\ ${ }^{6}$ Howard-Sloan-Koller Groep, Arnhem, Netherlands
}

\section{Edited by:}

Pinhas N. Dannon, Tel Aviv University, Israel

\section{Reviewed by:}

Landon Myer, University of Cape Town, South Africa

*Correspondence:

Patricia van Oppen, GGZ inGeest/VU Medical Center Academic Anxiety

Outpatient Clinic, A.J. Ernststraat 887;

10 Amsterdam, Netherlands.

e-mail:p.vanoppen@ggzingeest.nl
The Yale Brown Obsessive-Compulsive scale (Y-BOCS) is a semi-structured interview considered to be the gold standard in the measurement of obsessive-compulsive disorder (OCD) severity, yet findings about its factorial structure are conflicting. This study aimed at comparing different models, and testing whether factorial structure differs along various sub-groups. Exploratory and confirmatory factor analyses were conducted on Y-BOCS scores of a large OCD patient group $(n=544)$. A three-factor structure (obsessions, compulsions, and resistance) provided the best fit for the data across different factor analytic procedures. The difference in goodness of fit between the original two factor (obsessions and compulsions) and the three-factor solutions seemed, however, very small. Since the two-factor solution is the original theory-driven structure, and the most widely used, we recommend the use of this factor.

Keywords: obsessive-compulsive disorder, measurement, factor analysis

\section{INTRODUCTION}

The Yale Brown Obsessive-Compulsive Scale (Goodman et al., $1989 a, b)$ is regarded as the "gold standard" in the measurement of obsessive-compulsive disorder (OCD) symptom severity and treatment response (Steketee, 1994; Moritz et al., 2002). It is a semistructured interview that consists of 10 core items, 5 measuring time, interference, distress, resistance and control of obsessions (items: $1-5$ ), and 5 identical items measuring compulsions (items: 6-10). The items are rated from 0 (no symptoms) to 4 (severe symptoms), and yield a global severity score (range $0-40$ ). In addition, Goodman et al. (1989a) have suggested six investigational items (insight, avoidance, indecisiveness, pathological responsibility, pathological slowness, and pathological doubting). In contrast to the rest of the scale, the resistance items (items: 4,9 ) have demonstrated problematic psychometric properties and lower correlations to the Y-BOCS total scores (Goodman et al., 1989b; Woody et al., 1995). These findings have led to the suggestion of deleting the resistance items from the scale (Woody et al., 1995).

Since its introduction, nine publications investigated the factorial structure of the Y-BOCS, using either exploratory or confirmatory factor analysis. Contradictory results of these endeavors might explain the more common use of total scores in measuring treatment effects, rather than using the different suggested factors. The first study investigating the structure of the Y-BOCS involved an exploratory factor analysis (with a promax rotation) of 16 items (including the 6 investigational items), and concluded that all items load on a single factor (Fals-Stewart, 1992). This study was criticized by some researchers (Amir et al., 1997), who suggested that the inclusion of 6 items that are not computed into the total score may have obscured the differences between obsessions and compulsions by emphasizing the overall severity of each symptom, and that these findings although fitting the data may not be clinically pertinent. Consequent studies have almost exclusively involved the 10 core items of the Y-BOCS. Kim et al. (1994) conducted an exploratory factor analysis (with a varimax rotation) on the 10 item Y-BOCS, and found a three-factor structure emerged, with obsessions (items: 1, 2, 3, 5), compulsions (items: 6, 7, 8, 10) and resistance (items: 4,9 ). The resistance factor was the only factor not sensitive to medication effects in this study. These researchers suggested that contrary to the rest of the Y-BOCS, the resistance items measure components other than what was intended within the construct. This three-factor structure has been replicated by Moritz et al. (2002) using the same procedure, (exploratory factor analysis with a varimax rotation). In addition, they found that the resistance factor was sensitive to the effects of cognitive-behavioral therapy. The same study (Moritz et al., 2002) included an investigation of the factor structure of a 12 item version of the Y-BOCS. This version included the 10 usual items and 2 additional items assessing free intervals from obsessions and compulsions. They found that the 12-item version demonstrated an identical 3-factor structure, identical to the factor structure found by Kim et al. (1994). The longest interval free of obsessions and compulsions appeared to load on the obsessions and compulsions factors respectively. The 12-item version of the Y-BOCS, however, has not gained much attention from other researchers. An analysis of the psychometric properties of these items (Woody et al., 1995) has resulted in the conclusion that these two experimental items are largely redundant with existing items, and could be eliminated. 
McKay et al. (1995) conducted the first confirmatory factor analysis, and found the best fit to be a two-factor structure (obsessions: items 1, 2, 3, 4, 5 and compulsions: items 6, 7, 8, 9, 10). This study was criticized (Amir et al., 1997) because of its relatively small sample size and because the authors did not replicate their findings in a second sample, limiting the generalizability of their findings. Amir et al. (1997) used the largest sample to date $(n=404)$ and split it to two sub-samples, using confirmatory factor analysis. The best fit was obtained for a new two-factor structure (disturbance: items $2,3,7,8$; and symptom severity: items: $1,4,5,6,9,10)$. This new model was derived from clinical observations that OCD patients often do not clearly separate their OCD symptoms into obsessions and compulsions. Instead, they focus on the consequences of their disorder.

McKay et al. (1998) have conducted another confirmatory factor analysis and found parallel support for two-factor structures: (1) obsessions/compulsions, and (2) disturbance/symptom severity. These findings were consistent with earlier findings of factor structures detected by the confirmatory factor analytic method. Arrindel et al. (2002) achieved slightly different results (using confirmatory factor analysis), and the only model fitting the data was the obsessions/compulsions model (the disturbance and symptom severity factors exhibited a correlation of $r=0.93$, indicating that no reliable distinction whatsoever could be made between the factors). Similar results were obtained by Storch et al. (2005), where the original obsessions/compulsions model produced the best fit using confirmatory factor analysis. Finally, Deacon and Abramowitz (2005) have examined all existing Y-BOCS models with confirmatory factor analysis. No model provided a good fit for the data. As a consequence, they conducted exploratory factor analysis on the same sample and found yet a new factor structure: severity (items: $1,2,3,6,7,8)$, and resistance/ control $(4,5,9,10)$.

Several methodological differences and shortcomings in factorial studies of the Y-BOCS might explain the inconsistencies found. First, sample sizes of most investigations were rather small, relative to the statistical analyses applied (Comrey and Lee, 1992). Two studies using exploratory factor analysis (Kim et al., 1994; Moritz et al., 2002) have used a varimax rotation. This rotation assumes that relatively orthogonal constructs are being measured; an assumption that contradicts most research conducted thus far (Deacon and Abramowitz, 2005). Both studies have provided support for the three-factor model. However, control items (items: 5, 10) exhibited double loadings on the resistance factor as well as on the obsessions and compulsions factor. Kim et al. (1994) provided little reference to this problem, while Moritz et al. (2002), suggested that the ability to control symptoms is a joint function of the ability to resist the execution of compulsion, the emergence of obsessions, or the extent to which obsessions and compulsions are present. Furthermore, Moritz et al. (2002) have conducted confirmatory factor analysis on the same sample, and were the only investigators who found confirmation for this structure. However, they modeled control items both on the resistance factor and on the obsessions and compulsions factors. This is in contrast with their suggestion of fitting these items within the resistance factor only. Deacon and Abramowitz (2005) have found a different factor structure using an oblique rotation. Double loadings of control items may account particularly for the inconsistencies found using confirmatory factor analysis, whereas the use of an oblique vs. an orthogonal rotation may account for differences in exploratory factor analysis. Furthermore, a difficulty in comparing results of confirmatory factor analyses is that most studies have tested only a partial selection of existing models. Another limitation is that most publications provide limited sample characteristics, making it difficult to compare the samples used. Table 1 depicts all studies on factorial structure of the Y-BOCS, Y-BOCS version used, analytic procedures that were used, factor structures found, sample size and estimation of power according to Comrey and Lee's (1992) view, sample's gender ratio, average current age, and average age of onset.

The present study was designed to test which model provides the best structure for the Y-BOCS, using a large sample of OCD patients. In order to increase generalizability, the sample was split in two and parallel exploratory and confirmatory factor analyses were conducted. Since resistance items have proved to bring unstable factors, analyses of internal consistency were conducted, without these items, to ascertain whether their removal might increase internal consistency.

\section{MATERIALS AND METHODS PARTICIPANTS AND MEASURES}

Five hundred and forty four OCD patients participated in this study. The inclusion criteria consisted of: (1) main diagnosis of OCD according to DSM-IV criteria, (2) a minimum age of 17 years, and (3) at least a 1-year duration at intake. Exclusion criteria included: (1) suffering from organic brain disease, or (2) comorbid mental retardation, or (3) comorbid psychotic disorder. The study was approved by the VU-University Medical Centre's Ethical Review Committee. All study subjects were included after written informed consent had been given.

Patients consisted of 203 males (37.3\%), 341 (62.7\%) females, with a mean age of $37.71(\mathrm{SD}=11.06)$. Mean years of education achieved was 12.17 ( $\mathrm{SD}=2.99)$. To create a large sample, patients were recruited from four separate research projects of three Dutch academic outpatient anxiety clinics: (1) 129 patients (54 males and 75 females) were recruited from an OCD treatment study at the GGZ Buitenamstel, Amsterdam. The diagnostic assessment was conducted with the Anxiety Disorder Interview Schedule (ADIS; Bouwman et al., 1987), (2) 184 patients (71 males, 113 females) were recruited from an OCD course and treatment study at the GGZ Buitenamstel, Amsterdam. The diagnostic assessment was conducted with the Structured Clinical Interview on DSM-IV axis I diagnoses (SCID-I; First et al., 1995), (3) 159 patients (57 males, 102 females) were recruited from a research project of the Groningen university. The diagnostic assessment was conducted with the mini international neuropsychiatric interview (MINI; Sheehan et al., 1998), and (4) 72 patients (21 males, 51 females) were recruited from a research project of the Nijmegen university. The diagnostic assessment was conducted with the ADIS (Bouwman et al., 1987).

One hundred ninety patients $(34.93 \%)$ presented with a comorbid anxiety disorder, 147 patients $(27.02 \%)$ presented with a comorbid mood disorder, 34 patients presented with a comorbid 


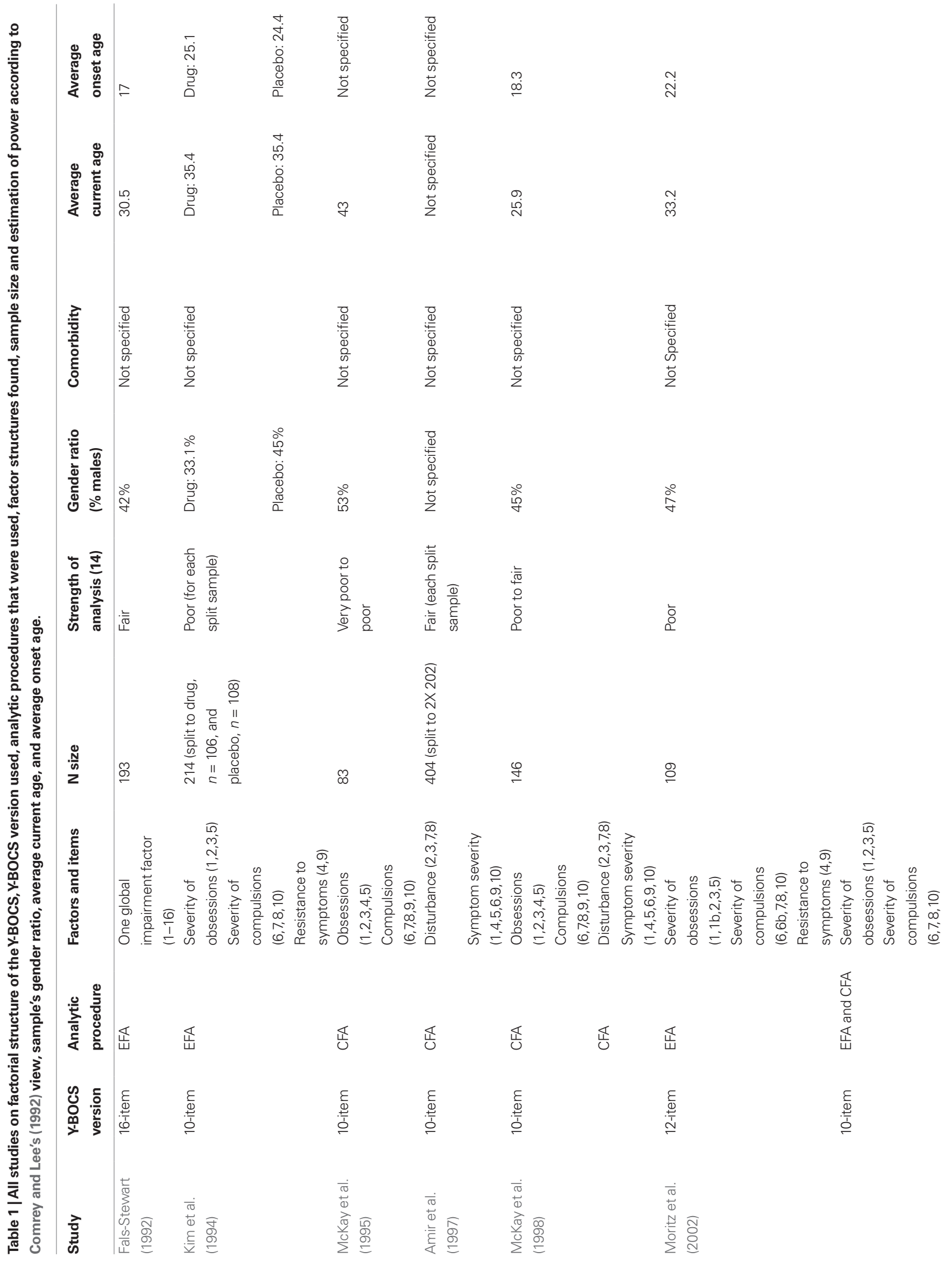




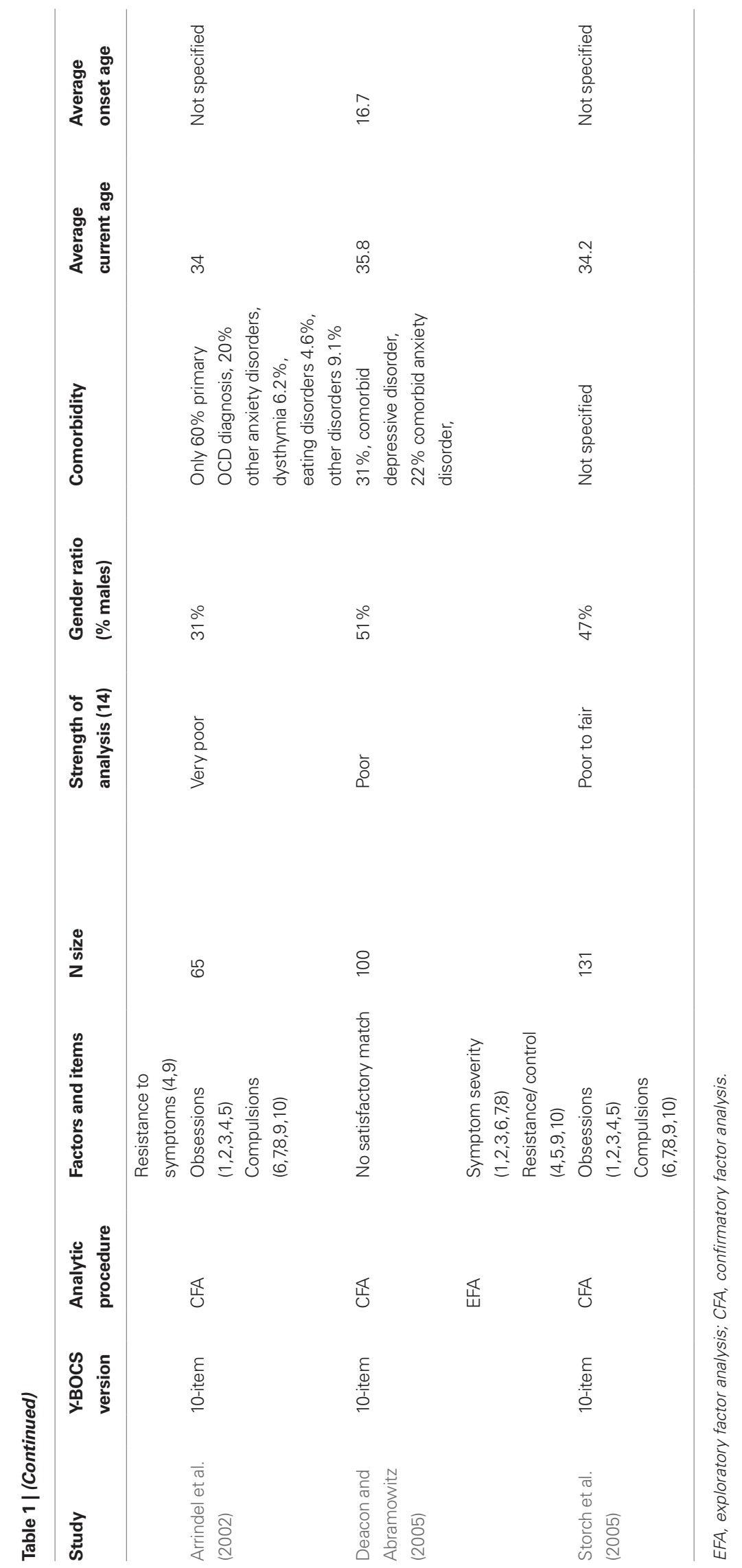


substance-related disorder (6.25\%), 29 patients (5.33\%) presented with a comorbid somatoform disorder, 12 patients $(2.21 \%)$ presented with a comorbid psychotic disorder, 9 patients presented with a comorbid eating disorder, 6 patients $(1.1 \%)$ were diagnosed with a disorder usually diagnosed in infancy, childhood or adolescence, 2 patients $(0.37 \%)$ presented with impulse control disorder not elsewhere classified, and 1 patient $(0.18 \%)$ presented with a comorbid gender identity disorder.

Since different assessment measures were used, it is important to note the implication of differences. The most comprehensive measure is the SCID-I, which includes most DSM-IV's Axis-I diagnoses. The MINI entails an assessment of anxiety disorders, mood disorders, somatoform disorders, substance-related disorders, and eating disorders. The ADIS entails assessment of anxiety disorders, mood disorders, eating disorders and substance-related disorders. At the Nijmegen study, an extended version of the ADIS was used, which also entails somatoform disorders.

The Y-BOCS (Goodman et al., 1989a,b), a semi-structured interview designed to measure symptom severity in OCD patients, was administered to all participants by trained psychologists or psychiatrics.

\section{DATA ANALYSES}

First, the sample was randomly split in two. On both samples, two sets of analyses were conducted: (1) Principal Components Analysis (PCA) exploratory factor analysis with an oblique (Promax) rotation, and (2) all proposed Y-BOCS factor structures were tested through confirmatory factor analysis (CFA, using LISRELL program 8.30 version; Jöreskog and Sörbom, 1993). CFA is a method to analyze the goodness of fit of a predetermined factor model to actual data. Following Amir et al. (1997), five commonly used fit indices were used: (1) chi-square, (2) adjusted goodness of fit index (AGFI), (3) root mean square error of approximation (RMSEA), comparative fit index (CFI), and (5) normed fit index (NFI). A non-significant Chi-square value indicates a good fit between a model and the data. AGFI values greater than 0.80 are considered a sign of good adjustment of the model to the data (Cole, 1987). RMSEA values of up to 0.05 indicate a close fit of the model to the data and values of up to 0.08 indicate a reasonable fit of the model to the data (Browne and Cudeck, 1993). CFI and NFI lie between 0 and 1, with larger values indicating better fit, and 1 representing optimal fit of the data to the model (Jöreskog and Sörbom, 1993). The logic for conducting both exploratory and confirmatory factor analyses on both samples was to establish the convergence between these two methods.

Second, Cronbach's alphas were calculated for the 10 and 8 item (without resistance items) versions of the Y-BOCS, to examine whether discarding resistance items results in increased internal consistency.

\section{RESULTS}

\section{FACTOR ANALYSES OF THE SPLIT SAMPLE}

First, exploratory factor analysis was conducted on the two randomly split samples (Table 2).

In the first sample, a three-factor structure emerged, with explained variance of the obsessions factor $=26.89 \%$, of the compulsions factor $=28.08 \%$, and of the resistance factor $=17.88 \%$.

Double loadings were found for items 5 (control over obsessions), and 9 (resistance to compulsions).

In the second sample, a similar three-factor structure emerged, with explained variance of the obsessions factor $=28.82 \%$, of the compulsions factor $=28.37 \%$, and of the resistance factor $=20.00 \%$.

Double loadings were found for items 5 (control over obsessions), and 10 (control over compulsions).

Then, confirmatory factor analyses were run on the two samples (Table 3).

In both samples, the best fit was achieved by the three-factor structure: obsessions, compulsions, and resistance.

\section{INTERNAL CONSISTENCY}

Cronbach's $\alpha$ (of the 10 item Y-BOCS $)=0.873 . \alpha(8$ items, without items 4, 9: the resistance items) $=0.879$.

Table 2 | Exploratory factor analysis of the first randomly split sample ( $n=272,104$ males, 168 females), and for the second randomly split sample ( $n=272,99$ males, 173 females).

\begin{tabular}{|c|c|c|c|c|c|c|}
\hline \multirow[t]{2}{*}{ Items } & \multicolumn{2}{|c|}{ Factor 1} & \multicolumn{2}{|c|}{ Factor 2} & \multicolumn{2}{|c|}{ Factor 3} \\
\hline & Sample 1 & Sample 2 & Sample 1 & Sample 2 & Sample 1 & Sample 2 \\
\hline 1. Obsessions (Time) & 0.87 & 0.86 & 0.34 & 0.40 & 0.17 & 0.25 \\
\hline 2. Obsessions (interference) & 0.86 & 0.88 & 0.41 & 0.48 & 0.18 & 0.28 \\
\hline 3. Obsessions (Distress) & 0.89 & 0.89 & 0.37 & 0.38 & 0.35 & 0.27 \\
\hline 4. Obsessions (Resistance) & 0.26 & 0.47 & 0.26 & 0.23 & 0.89 & 0.85 \\
\hline 5. Obsessions (Control) & 0.69 & 0.81 & 0.34 & 0.37 & 0.67 & 0.59 \\
\hline 6. Compulsions (Time) & 0.49 & 0.33 & 0.76 & 0.87 & 0.10 & 0.30 \\
\hline 7. Compulsions (interference) & 0.48 & 0.48 & 0.83 & 0.88 & 0.19 & 0.30 \\
\hline 8. Compulsions (Distress) & 0.26 & 0.48 & 0.83 & 0.83 & 0.36 & 0.38 \\
\hline 9. Compulsions (Resistance) & 0.10 & 0.12 & 0.58 & 0.47 & 0.69 & 0.84 \\
\hline 10. Compulsions (Control) & 0.26 & 0.36 & 0.84 & 0.81 & 0.44 & 0.63 \\
\hline
\end{tabular}

${ }^{*}$ Factor loadings of 0.5 and above are marked in bold. 
Table 3 | Confirmatory factor analysis of the first randomly split sample ( $n=272,104$ males, 168 females), and for the second randomly split sample ( $n=272,99$ males, 173 females).

\begin{tabular}{|c|c|c|c|c|c|c|c|c|c|c|c|}
\hline 1 & 35 & $\begin{array}{l}567.502 \\
<0.001\end{array}$ & $\begin{array}{l}618.542 \\
<0.001\end{array}$ & 0.474 & 0.413 & 0.237 & 0.248 & 0.594 & 0.626 & 0.606 & 0.638 \\
\hline 3 & 34 & $\begin{array}{l}293.396 \\
<0.001\end{array}$ & $\begin{array}{l}285.960 \\
<0.001\end{array}$ & 0.698 & 0.698 & 0.168 & 0.165 & 0.790 & 0.827 & 0.808 & 0.844 \\
\hline 4 & 34 & $\begin{array}{l}567.499 \\
<0.001\end{array}$ & $\begin{array}{l}616.408 \\
<0.001\end{array}$ & 0.459 & 0.392 & 0.241 & 0.241 & 0.594 & 0.594 & 0.605 & 0.605 \\
\hline
\end{tabular}

*1. One factor model (1-10).

2. Three-factor model: Severity of obsessions (items 1,2,3,5), Severity of compulsions (items 6, 7,8, 10), Resistance to symptoms (items 4,9).

3. Two-factor model: Obsessions (items 1,2,3,4,5), Compulsions (items 6,7,8,9,10). This is the current structure of the Y-BOCS.

4. Two-factor model Disturbance (items 2,3,7,8), Symptom severity (items 1,4,5,6,9,10).

5. Two-factor model Resistance/ control (items 4,5,9,10), Symptom severity (items 1,2,3,6, 7,8).

\section{DISCUSSION}

The purpose of the present study was to examine the factorial structure of the Y-BOCS using the largest patient sample so far, and examining factorial structure of different sub-groups for the first time. Exploratory factor analyses on both samples revealed an identical, three-factor solution, with obsessions, compulsions, and resistance factors. Confirmatory factor analyses run on the same sample produce identical findings. The three-factor solution entails the best fit to the data, though it does not fully fulfill the optimal requirement for fit indices used. Chi-square values remain highly significant, indicating that a significant proportion of the data is not explained by the proposed factor structure. AGFI indices fall short of the values recommended for a good fit, and RMSEA values fall above 0.08 , which again indicate significant differences between model and data, and NFI and CFI values also indicate less than desirable fit between model and data. Another interesting finding is that the differences between the goodness of fit of the three-factor model, and the original obsessions-compulsions two-factor model are minimal. Use of the two-factor model might solve the problem of double loadings of resistance and control items in the three-factor model, thus better differentiate between factors. Since the two-factor solution is the most widely used, and compatible with original theoretical and DSM-IV conceptualizations of OCD, it seems that keeping this model is best advised.

Reliability analyses revealed that internal consistency of the Y-BOCS does not improve without the Y-BOCS' resistance items. This is an interesting finding considering current cognitive models of OCD, which view particularly the attempt to resist obsessions as an etiological factor in the development and maintenance of OCD (Obsessive-Compulsive Cognitions Working Group, 1997). Higher levels of resistance are viewed according to these models as leading to paradoxical effects of thought suppression, and therapeutic efforts are directed at diminishing resistance rather than increasing it. Yet, our findings suggest that both resistance items are well related to the rest of the Y-BOCS' items. Therefore, findings on the current sample suggest that there is no justification in discarding resistance items. Nevertheless, it is interesting to note that exploratory factor analyses without resistance items produce a two-factor structure, without double loadings of items. This exemplifies the contribution of resistance items to the unstableness of the Y-BOCS factor structure.

Strengths of this investigation include the use of a very large patient sample, so that reasonable statistical power has been achieved. Weaknesses include the use of various assessment measures for diagnosis, which might have particularly influenced comorbidity rates. Further, since data was derived from various research projects, no comparison with other OCD measures was available.

Using a large sample we have demonstrated that the three-factor model with obsessions, compulsions, and resistance items best fits our data. The two-factor obsessions-compulsions factor, however, possesses almost identical goodness of fit to the data, yet presents some clear advantages in being the original theory-derived structure and being more widely used. We therefore recommend subsequent use of the Y-BOCS in its current two-factor. 


\section{REFERENCES}

Amir, N., Foa, E. B., and Coles, M. E. (1997). Factor structure of the YaleBrown Obsessive Compulsive Scale. Psychol. Assess. 9, 312-316.

Arrindel, W.A., de Vlaming, I., Eisenhardt, B. M., van Berkum, D. E., and Kwee, M. G.T. (2002). Cross-cultural validity of the Yale-Brown Obsessive Compulsive Scale. J. Behav. Ther. Exp. Psychiatry 33, 159-176.

Bouwman, T.K.,Scholing,A.,Emmelkamp, P. M. G., and Dijkstra, W. (1987). Anxiety Disorders Interview Schedule (ADIS, intern report). Groningen: Department of Clinical Psychology, University of Groningen.

Browne, M. W., and Cudeck, R. (1993). "Alternative ways of assessing model fit," in Testing Structural Equation Models, eds K. A. Bollen and J. S. Long (Thousand oaks, CA: Sage), pp. 136-162.

Cole, D.A. (1987). Utility of factor analysis in test validation research. J. Consult. Clin. Psychol. 55, 584-594.

Comrey, A. L., and Lee, H. B. (1992). A First Course in Factor Analysis (2nd Edn). Hillsdale, NJ: Lawrence Erlbaum Associates.

Deacon, B. J., and Abramowitz, J. S. (2005). The Yale-Brown Obsessive Compulsive Scale: factor analysis, construct validity, and suggestions for refinement. J. Anxiety Disord. 19, 573-585.
Fals-Stewart, W. (1992). A dimensional analysis of the Yale-Brown Obsessive Compulsive Scale. Psychol. Rep. 70, 239-240.

First, M. B., Spitzer, R. L., Gibbon, M., and Williams, J. B. (1995). Structured Clinical Interview for DSM-IV (SCID). NY, New York: Psychiatric Institute, Biometrics Research.

Goodman, W. K., Price, L. H., Rasmussen, S. A., Mazure, C., Fleischmann, R., Hill, C. L., Heninger, G. R., and Charney, D. S. (1989a). The Yale-Brown ObsessiveCompulsive Scale I: development, use, and reliability. Arch. Gen. Psychiatry 46, 1006-1011.

Goodman, W. K., Price, L.H., Rasmussen, S.A., Mazure, C., Delgado, P., Heninger, G. R., and Charney, D. S. (1989b). The Yale-Brown Obsessive Compulsive Scale: II validity. Arch. Gen. Psychiatry $46,1012-1016$.

Jöreskog, K. G., and Sörbom, D. (1993). LISREL VIII: User's Reference Guide. Chicago: Scientific Software.

Kim, S. W., Dysken, M. W., Pheley, A. M., and Hoover, K. M. (1994). The YaleBrown Obsessive Compulsive Scale: measures of internal consistency. Psychiatry Res 51, 203-211.

McKay, D., Danyko, S. C., Neziroglu, F., and Yaryura-Tobias, J. A. (1995). Factor structure of the Yale-Brown Obsessive-Compulsive Scale: a two dimensional measure. Behav. Res. Ther. 33, 865-869.
McKay, D., Neziroglu, F., Stevens, K., and Yaryura-Tobias, J.A. (1998). The YaleBrown Obsessive-Compulsive Scale: Confirmatory factor analytic findings. J. Psychopathol. Behav. Assess. 20, 265-274.

Moritz, M., Meier, B., Klos, M., Jacobsen, D., Wein, C., Fricke, S., and Hand, I. (2002). Dimensional structure of the Yale-Brown Obsessive-Compulsive Scale (Y-BOCS). Psychiatry Res. 109, 193-199.

Obsessive Compulsive Cognitions Working Group. (1997). Cognitive assessment of obsessive-compulsive disorder. Behav. Res. Ther. 35 667-681.

Sheehan, D. V., Lecrubier, Y., Sheehan, K. H., Amorim, P., Janavs, J., Weiller, E. Hergueta, T., Baker, R., and Dunbar, G. C. (1998). The Mini-International Neuropsychiatric Interview (M.N.N.I.): the development and validation of a structured diagnostic psychiatric interview for DSM-IV and ICD-10. J. Clin. Psychiatry 59(suppl. 20), 22-33.

Steketee, G. (1994). Behavioral assessment and treatment planning with obsessive-compulsive disorder: a review emphasizing clinical application. Behav. Ther. 25, 613-633.

Storch, E. A., Shapira, N. A., Dimoulas, E., Geffken, G. R., Murphy, T. K., and Goodman, W. K. (2005). YaleBrown obsessive-compulsive scale: the dimensional structure revisited. Depress. Anxiety 22, 28-35.

Woody,S.R.,Steketee, G., and Chambeless, D. L. (1995). Reliability and validity of the Yale-Brown ObsessiveCompulsive Scale. Behav. Res. Ther. 33, 597-605.

Conflict of Interest Statement: The authors declare that the research was conducted in the absence of any commercial or financial relationships that could be construed as a potential conflict of interest.

Received: 18 March 2010; paper pending published: 19 April 2010; accepted:01 June 2010; published online: 15 July 2010.

Citation: Anholt GE, van Oppen P, Cath DC, SmitJH, den Boer JA, Verbraak MJPM and van Balkom AJLM (2010) The Yale Brown Obsessive-Compulsive scale: factor structure of a large sample. Front. Psychiatry 1:18. doi: 10.3389/fpsyt.2010.00018 This article was submitted to Frontiers in Impulsivity, Compulsivity and Behavioral Dyscontrol, a specialty of Frontiers in Psychiatry.

Copyright (C) 2010Anholt, van Oppen, Cath, Smit, den Boer, Verbraak and van Balkom. This is an open-access article subject to an exclusive license agreement between the authors and the Frontiers Research Foundation, which permits unrestricted use, distribution, and reproduction in any medium, provided the original authors and source are credited. 